\title{
Dolor sacroiliaco
}

a articulación sacroiliaca (ASI) es una causa posible de dolor lumbar. Sin embargo sus características anatómicas y biomecánicas hacen difícil conocer la verdadera prevalencia de procesos dolorosos de la ASI, así como realizar su evaluación y diagnóstico. Una vez descartados los procesos específicos, inflamatorios (espondiloartritis), infecciosos, fracturas osteoporóticas o tumorales, la mayoría de los procesos no específicos se engloban bajo la entidad denominada disfunción de la articulación sacroiliaca (DASI).

La disfunción de la ASI ha sido definida como dolor localizado en la región glútea, reproducible por el estrés y pruebas de provocación de la articulación, y se alivia de forma fiable por bloqueo selectivo anestésico de la ASI.

La ASI es una articulación diartrodial única que une las caras planares e irregulares del sacro y los iliacos, con presencia de cartílago hialino y también fibrocartílago, recubierta en sus $2 / 3$ inferiores de sinovial y un capsula fibrosa. Está fijada por múltiples estructuras ligamentosas, que limitan prácticamente su movilidad. Si bien los movimientos de la articulación sacroiliaca son pequeños en magnitud, son de naturaleza compleja: la rotación media puede llegar a ser de $1,2^{\circ}$ a $3^{\circ}$ y la media de translación de 0,7 a $2 \mathrm{~mm}$, aunque varían considerablemente con la morfología de cada individuo. La mujer tiene mayor laxitud por efecto hormonal.

Un dolor en la parte lumbar y las nalgas puede tener su origen en patología de la ASI, pero también puede irradiarse a la extremidad inferior. Los estudios de mapeo de dolor durante la inyección forzada en la ASI han producido resultados variables. Adicionalmente la inervación también es compleja con origen en diferentes raíces, fundamentalmente S1 y S2 y ramas de del N, obturador y glúteo posterior que podría dar lugar a dolor referido glúteo. El dolor lumbar/glúteo de origen en ASI parece tener una prevalencia posible del 16 al $30 \%$ según algunos estudios.

El problema fundamental de esta patología es su diagnóstico. Las diferentes maniobras clínicas de provocación tienen una baja sensibilidad y especificidad. Aunque no se conoce bien la utilidad de combinar varias de ellas. Las pruebas de imagen, sin patología específica son poco útiles. La gammagrafía ósea tiene un alto grado de falsos positivos, y las técnicas de bloqueo diagnostico sólo permiten identificar el 60 de los casos con doble bloqueo, y en muchos casos hay extravasación del anestésico que puede dar lugar a confusión.

En este número de la Revista de la Sociedad Española del Dolor, los Dres. Juan Carlos Acevedo y Silvia Quintero, del Hospital Universitario San Ignacio de Bogotá (Colombia), aportan un interesante estudio piloto que evalúa la utilización de un cuestionario clínico que combina características del dolor lumbar, la positividad de 3 maniobras de exploración de la ASI y la positividad de la gammagrafía ósea (con una puntuación total de 0 a 5, 1 punto por cada ítem positivo). Dicho cuestionario es el resultado de una revisión de la bibliografía sobre pruebas diagnósticas del dolor SI y la selección hecha por los autores de los estudios que han considerado más válidos. La fiabilidad de este cuestionario es comprobada en 68 pacientes con dolor lumbar de diversas patologías (enfermedad facetaría 33, radiculopatía 25, disfunción sacroilica 6 y otras causas 4). Las puntuaciones de los casos de DASI son 4 o 5 puntos. Pero las puntaciones del cuestionario 
se superponen entre los diferentes diagnósticos. Así, un $40 \%$ de los pacientes con enfermedad facetaria y un $36 \%$ de radiculopatías tienen puntuaciones de 4 o 5 en el cuestionario.

Sin duda hay aspectos metodológicos que se deben mejorar, como los criterios estrictos de selección de los grupos control y casos, el número de pacientes necesario en cada grupo (sólo 6 casos de DSAI), y un diseño de un cuestionario con mayor número de ítems a evaluar, para luego hacer la selección de aquella combinación que mejores datos estadísticos ofrezca.

Sin embargo la importancia de estudio es la filosofía original de intentar encontrar una herramienta útil y sencilla, combinando criterios clínicos, exploratorios y radiológicos que ayude al diagnóstico y tratamiento de esta difícil patología (posiblemente por su heterogeneidad). Este es un camino que debemos seguir y perfeccionar y en el que esperamos que los autores continúen aportando avances de mayor solidez.

J. Vidal Fuentes

Unidad de Dolor Reumático. Hospital Universitario de Guadalajara

\section{BIBLIOGRAFÍA}

1. Forst SL, Wheeler MT, Fortin JD, Vilensky JA. The sacroiliac joint: anatomy, physiology and clinical significance. Pain Physician 2006;9:61-7.

2. Rupert MP, Lee M, Manchikanti L, Datta S, Cohen SP. Evaluation of sacroiliac joint interventions: A systematic appraisal of the literature. Pain Physician 2009;12:399-418.

3. Simopoulos TT, Manchikanti L, Singh V, Gupta S, Hameed H, Diwan S, et al. A systematic evaluation of prevalence and diagnostic accuracy of sacroiliac joint interventions. Pain Physician 2012;15:E305-44.

4. Berthelot JM, Labat JJ, Le Goff B, Gouin F, Maugars Y. Provocative sacroiliac joint maneuvers and sacroiliac joint block are unreliable for diagnosing sacroiliac joint pain. Joint Bone Spine 2006;73:17-23.

5. Van der Wurff P, Meyne W, Hagmeijer RH. Clinical tests of the sacroiliac joint. Man Ther 2000;5:89-96. 